\title{
The clinical course of actinic keratosis correlates with underlying molecular mechanisms
}

A. Bakshi, ${ }^{1,2}$ R. Shafi, ${ }^{1}$ J. Nelson, ${ }^{1}$ W.C. Cantrell, ${ }^{1}$ S. Subhadarshani (iD, ${ }^{1}$ A. Andea, ${ }^{3}$ M. Athar ${ }^{1,4}$ and C.A. Elmets ${ }^{1,4,5}$

${ }^{1}$ Department of Dermatology and ${ }^{4} O^{\prime}$ Neal Comprehensive Cancer Center, University of Alabama at Birmingham, Birmingham, AL, U.S.A.

${ }^{2}$ Rutgers New Jersey Medical School, New Brunswick, NJ, U.S.A.

${ }^{3}$ Department of Pathology, University of Michigan, Ann Arbor, MI, U.S.A.

${ }^{5}$ Birmingham VA Medical Center, Birmingham, AL, U.S.A.

Linked Comment: O'Shaughnessy. Br J Dermatol 2020; 182:834-835

Correspondence

Craig Elmets.

E-mail: celmets@uabmc.edu

\section{Accepted for publication}

7 July 2019

\section{Funding sources}

This work was supported by National Institutes of Health grants P01CA210946, R01CA193885, P30 CA013148, N01 CN2012-00033 and RO1ESOO26219; Veterans Administration grant $101 \mathrm{BX} 003395$.

\section{Conflicts of interest}

None to declare.

A.B. and R.S. contributed equally.

DOI 10.1111/bjd. 18338

\section{Summary}

Background Actinic keratoses (AKs) are common premalignant skin lesions triggered by excessive ultraviolet exposure. The majority of AKs regress or persist, but some progress to squamous cell carcinomas. Biomarkers associated with their persistence, progression and regression have not been characterized.

Objectives We performed skin biopsies in patients with extensive actinic damage to identify biomarkers that correlate with clinical progression and regression of AKs. Methods This was an observational study of a cohort of patients with extensive actinic damage. AKs were mapped on a clear plastic template in 26 patients at months 3, 6, 9 and 11. Biopsies were taken from randomly selected, predetermined AKs and were evaluated for p53, E-cadherin, Snail, Slug and Twist. The study is registered at Clinicaltrials.gov: NCT00027976.

Results p53 exhibited greater expression in clinically apparent AKs (histological score $2.89 \pm 1.45)$ than in regressed AKs $(0.75 \pm 0.96) ; \mathrm{P}<0.01$. There was also significantly less membrane E-cadherin, the lack of which is a marker of epithelial-mesenchymal transition, in clinically apparent AKs $(1.89 \pm 1.81)$ than in sun-exposed skin $(3.07 \pm 1.75) ; \mathrm{P}<0.005$. The E-cadherin transcription repressors Snail, Slug and Twist were increased in AKs compared with sun-exposed skin. A limitation of the study is that measurement of histological biomarkers was not a primary end point. In addition, patients were allowed to apply sunscreens.

Conclusions At the molecular level, loss of E-cadherin and an increase in p53 are linked to the dynamic interplay between the persistence, progression and regression of AKs.

\section{What's already known about this topic?}

- Actinic keratoses (AKs) are common dysplastic epidermal lesions that result from chronic and excessive ultraviolet exposure.

- Biomarkers associated with progression and regression of AK have not been characterized.

\section{What does this study add?}

- Decreased E-cadherin and increased p53, Snail, Slug and Twist (E-cadherin transcription factors) were associated with progression from AK to nonmelanoma skin cancer. 


\section{What is the translational message?}

- Strategies targeting these molecules may be effective in reversing rising skin cancer rates.

- E-cadherin, p53, Snail, Slug and Twist are potential biomarkers that may be used to assess the efficacy of existing chemopreventive agents.

Actinic keratoses (AKs), also known as solar keratoses, are common dysplastic epidermal lesions that result from chronic and excessive ultraviolet (UV) exposure. Clinically, they range from small, flesh-coloured, erythematous papules to large plaques with yellow or brown scales and crusts that appear on sun-exposed areas of the face, scalp and extremities. ${ }^{1,2}$ In individuals with multiple AKs, the cumulative lifetime risk of developing an invasive squamous cell skin cancer is as high as 6-10\%. ${ }^{3}$ Histologically, AKs are composed of atypical keratinocytes characterized by alternating areas of parakeratosis and orthokeratosis, a loss of polarity, pleomorphic or hyperchromatic nuclei and an inflammatory infiltrate comprised of lymphocytes or plasma cells.

AKs are more frequent in individuals over the age of 45 years, due to escalating photodamage from cumulative sun exposure. $^{4,5}$ Other risk factors include lower Fitzpatrick sun reactive skin types, residence in lower latitudes, male sex, baldness, a prior history of skin cancer and an immunocompromised state. ${ }^{4,6-8}$ Management of actinically damaged skin represents a considerable burden to the healthcare systems of many countries worldwide. ${ }^{5}$ Based on U.S. health claims data, estimates are that over 10 million individuals in the U.S.A. are evaluated and treated annually for actinically damaged skin, at a cost of U.S. $\$ 1.68$ billion. ${ }^{5}$

The p53 tumour suppressor gene controls cell proliferation, regulates apoptosis and facilitates DNA repair by interrupting cell-cycle progression. p53 mutations are the most common genetic alteration associated with the development of various types of cancers including squamous cell carcinoma (SCCs). ${ }^{9,10}$ Chromosomal p53 mutations are present in $90 \%$ of SCCs and up to $100 \%$ of AKs. ${ }^{9}$

E-cadherin is an intercellular adhesion molecule that plays a significant role in maintaining epithelial architectural integrity. E-cadherin repression permits nonmotile epithelial cells to transform into motile mesenchymal-like cells through a process known as epithelial-mesenchymal transition. ${ }^{11,12}$ The resulting cellular phenotype is characterized by loss of intercellular bonds and an increase in migrational ability, leading to tumour invasion. ${ }^{13}$ E-cadherin expression is a highly regulated process, and the E-cadherin transcription repressors Snail, Slug and Twist play an important regulatory role in epithelialmesenchymal transition. ${ }^{14}$

The purpose of this study was to investigate the natural history of AKs in patients with extensive actinic damage and to determine whether biomarkers might predict which AKs are likely to progress to keratinocyte cancers (KCs) and which are likely to regress back to non-AK-bearing, sun-exposed skin. KCs are also known as nonmelanoma skin cancers (NMSCs), and the two terms are used interchangeably in this manuscript.

\section{Materials and methods}

\section{Actinic keratosis mapping}

This was an observational study of a cohort of patients with extensive actinic damage. We used data and biopsy specimens collected from 26 participants in the celecoxib NMSC chemoprevention trial who were randomized to receive placebo. ${ }^{15}$ Only samples from patients on placebo were included in the study. Approval for the study was obtained prior to its initiation from the University of Alabama at Birmingham (UAB) institutional review board. Individuals at high risk for NMSC were eligible to participate if they were over 18 years of age, had an extensive number of AKs and had Fitzpatrick skin types I, II or III. To be qualified as high risk, patients were required to have 10-40 AKs on the upper extremities, neck, face and scalp along with prior histological evidence of at least one AK or NMSC. ${ }^{15}$

Participants were instructed to abstain from topical prescription and over-the-counter medications; however, moisturizers, emollients and sunscreens were allowed. The patients were evaluated for AKs at 0, 3, 6, 9 and 11 months. A study investigator marked the location of each AK on a clear plastic template that was placed over the upper extremities, neck, face and scalp. Lesions with characteristic scaling, keratotic patches, erythema and sandpaper-like scale were identified as AKs. ${ }^{15}$ The study investigators used different plastic templates to mark AKs at each visit and were blinded to the template results from previous visits. At the end of the study, AK templates from months $0,3,6,9$ and 11 were compared to assess the number of new, persistent and regressed AKs.

\section{Skin biopsies and histological grading}

At baseline, two AKs on the upper extremities were randomly selected for biopsy, one of which was removed at baseline and the other at month 9. If, at month 9, an AK selected for biopsy was no longer present clinically, the site at which it had been located (i.e. regressed AK) was still biopsied. AK biopsies were evaluated based on the degree of proliferation and severity of atypia.

Each component in this grading scheme was given a score of 1-3 (Table 1). Nodular basal cell carcinoma (BCC) and well-differentiated SCC samples were obtained from the UAB tissue bank and were not from the patients who had been followed for 11 months. 
Table 1 Histological grading scheme

\begin{tabular}{lll}
\hline Atypia & Proliferation & Score \\
\hline$<1 / 3$ epidermal & No downward budding & 1 \\
$\begin{array}{l}\text { involvement } \\
1 / 3 \text { to } 2 / 3 \text { epidermal } \\
\text { involvement }\end{array}$ & Focal downward budding & 2 \\
$2 / 3$ to full epidermal & $\begin{array}{c}\text { Extensive downward budding } \\
\text { involvement }\end{array}$ & 3 \\
\hline
\end{tabular}

\section{Biomarker expression}

Biomarker expression was graded based on staining intensity and the continuity of expression. For p53, Snail, Slug and Twist this was 0 , no staining; 1 , minimal; 2 , mild; 3 , moderate; 4 , strong; 5 , very strong; and for E-cadherin: 0, no staining; 1-2 minimal; 3-4, mild; 5-6, moderate; 7-8, strong; 9-10, very strong.

\section{Immunofluorescence staining}

Paraffin tissue sections after deparaffinization and rehydration were processed further for immunofluorescence staining. Nonspecific antibody binding was blocked with $2 \%$ bovine serum albumin. After overnight incubation with primary antibodies to E-cadherin and p53 (Santa Cruz Biotechnology, Dallas, TX, U.S.A.), the tissue sections were incubated with a fluorescence-coupled secondary antibody and visualized using fluorescence microscopy.

\section{Immunohistochemical staining}

The paraffin-embedded tissue sections were deparaffinized and rehydrated. The antigen unmasking was done with $10 \mathrm{mmol}$ $\mathrm{L}^{-1}$ citrate buffer ( $\mathrm{pH} 6.0$ ) and the nonspecific antigen binding was blocked by incubation with $2 \%$ bovine serum albumin. The sections were incubated overnight at $4{ }^{\circ} \mathrm{C}$ with primary antibodies to Snail, Slug and Twist (Santa Cruz Biotechnology). This was followed by incubation with peroxidase-coupled secondary antibody and, finally, visualization with 3,3'-diaminobenzidine substrate.

\section{Statistical analysis}

The primary objective of this analysis was to assess whether there are biomarkers that can correlate with progression to skin cancer. The secondary objective was to determine the natural history of AKs. An independent Student's t-test was conducted to compare biomarker expression grading scores among the various tissue sample groups.

\section{Results}

Twenty-six white patients with extensive actinic damage on the upper extremities, neck, face and scalp were enrolled in the study. Table 2 summarizes the patient characteristics. In
Table 2 Patient characteristics

\begin{tabular}{lc}
\hline Characteristic & Number of patients \\
\hline Age range (years) & 1 \\
$30-39$ & 2 \\
$40-49$ & 7 \\
$50-59$ & 8 \\
$60-69$ & 8 \\
$70-79$ & \\
Fitzpatrick skin type & 4 \\
I & 16 \\
II & 6 \\
III & \\
Sex & 19 \\
Male & 7 \\
Female & \\
\hline
\end{tabular}

total 610 AKs were present at baseline, with a mean \pm SD of $23.5 \pm 7.9$ AKs per person. The total number of lesions remained steady over the 11-month observation period (Table 3). At 11 months there was a mean increase of only $1.7 \pm 11.8$ AKs per patient from baseline, which was not statistically significant. This is because over the 11 months, a nearly equivalent number of new AKs appeared as regressed. Overall, $43 \%$ clinically regressed without recurrence, while 87 $(14 \cdot 3 \%), 52(8 \cdot 5 \%), 42(6 \cdot 9 \%)$ and $82(13 \cdot 4 \%)$ regressed at $3,6,9$ and 11 months, respectively. The mean time to regression was 6.2 months. On the other hand, $32 \cdot 6 \%$ of AKs present at baseline regressed and recurred at one of the follow-up visits; $24 \%$ never regressed, $8 \%$ regressed twice and $1 \%$ regressed twice and recurred twice.

\section{Histological features of actinic keratoses}

There were 18 pairs of biopsies evaluated at baseline and at 9 months, for a total of 36 biopsies. Of the 36 AKs clinically present and biopsied, five were not confirmed as AKs based on histological analysis (lichen simplex chronicus or solar lentigines). Five of 18 of the 9-month biopsies had regressed and did not show histological evidence of AK. There was not a statistically significant change in the degree of proliferation or atypia of the AKs over the 9-month period (Fig. 1).

\section{Biomarker analysis}

Because changes in mutant p53 and E-cadherin are associated with SCC development in animal models, ${ }^{9,11,16}$ we sought to

Table 3 Number of actinic keratoses (AKs) present on each patient

\begin{tabular}{lll}
\hline Timepoint & AKs per patient, mean \pm SD & Total no. of AKs \\
\hline Baseline & $23 \cdot 5 \pm 7 \cdot 9$ & 610 \\
Month 3 & $25 \cdot 8 \pm 13 \cdot 1$ & 671 \\
Month 6 & $23 \cdot 1 \pm 11 \cdot 6$ & 600 \\
Month 9 & $27 \cdot 8 \pm 16 \cdot 9$ & 723 \\
Month 11 & $25 \cdot 0 \pm 16 \cdot 6$ & 650 \\
\hline
\end{tabular}




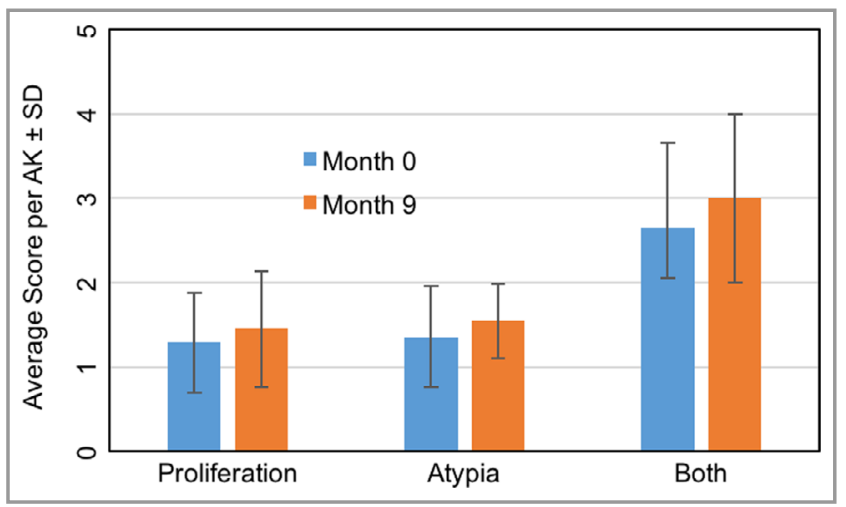

Fig 1. Histological score for the atypia and proliferation severity of randomly selected actinic keratoses (AKs) at baseline and when biopsied at 9 months. At baseline vs. month 9: $\mathrm{P}=0.52$ for proliferation, $\mathrm{P}=0.44$ for atypia and $\mathrm{P}=0.37$ for atypia and proliferation (both).

determine whether there was a change in p53 and/or E-cadherin expression when sun-protected skin, chronically sundamaged skin and AKs were compared. AKs and sun-exposed skin both expressed p53. Significantly reduced p53 was observed in regressed AKs and non-sun-exposed skin samples compared with clinically apparent AKs and sun-exposed skin (Fig. 2). BCC and SCC specimens were not collected from the patients enrolled in the study. Therefore, representative

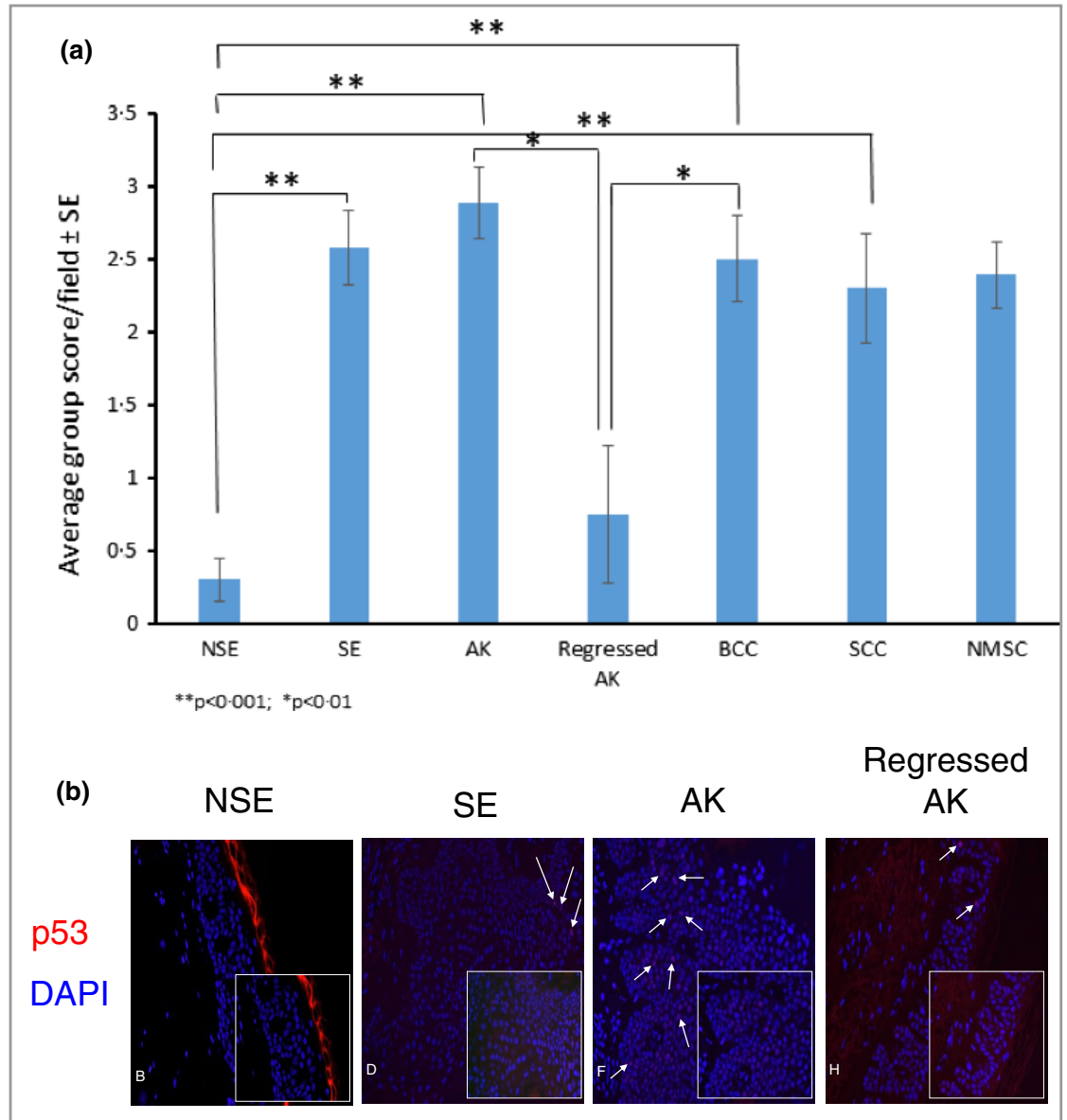

Fig 2. (a) Average nuclear expression score of p53 in skin biopsy samples: non-sun-exposed skin (NSE), sun-exposed skin (SE), clinically present actinic keratosis (AK), regressed AK, basal cell carcinoma (BCC), squamous cell carcinoma (SCC) and combined BCC and SCC (i.e. nonmelanoma skin cancer, NMSC). (b) Immunofluorescence of p53 with 4',6-diamidino-2-phenylindole (DAPI). The arrows depict nuclear expression of p53. 
samples were taken from the UAB tissue bank of cutaneous diseases. Those tissues showed enhanced expression of p53 relative to non-sun-exposed skin.

As E-cadherin is associated with progression of skin cancers in animal models, ${ }^{11}$ sun-protected skin, chronically sundamaged skin, AKs and KCs were analysed for E-cadherin expression. There was a progressive decline from non-sunexposed skin to sun-exposed skin to AKs (Fig. 3). E-cadherin expression in BCC and SCC was similar to that in AKs, suggesting at this point that it may be more of a marker of AK persistence rather than progression to KCs. In sites in which AKs had regressed, E-cadherin expression was less, although not significantly, than in AKs that had not regressed (Fig. 3).

E-cadherin is carefully regulated by the transcription repressors Snail, Slug and Twist. ${ }^{16}$ We investigated whether these proteins might be altered in actinically damaged skin. Sunexposed skin, AK, BCC and SCC expressed significantly greater levels of Snail, Slug and Twist than non-sun-exposed skin
(Fig. 4). In addition, expression levels of these proteins were significantly reduced in regressed AK samples compared with sun-exposed skin, AK and BCC (Fig. 4).

\section{Discussion}

AKs are common preneoplastic lesions that are precursors and risk factors for cutaneous SCCs. ${ }^{1,6,7,17-20}$ Their prevention and treatment reduce the significant likelihood of developing one or more KCs. ${ }^{1}$ We found that the appearance and regression of AKs is a dynamic process. The same AKs that were present at one time were absent at another, but had the capacity to reappear later. Over $40 \%$ of AKs identified at baseline regressed over the 11-month observation period, 33\% recurred, $11 \%$ regressed twice and a small number reappeared twice. As a result, the aggregate number of AKs over 11 months remained relatively constant. Moreover, there was no change in the histological severity of the AKs biopsied at 9

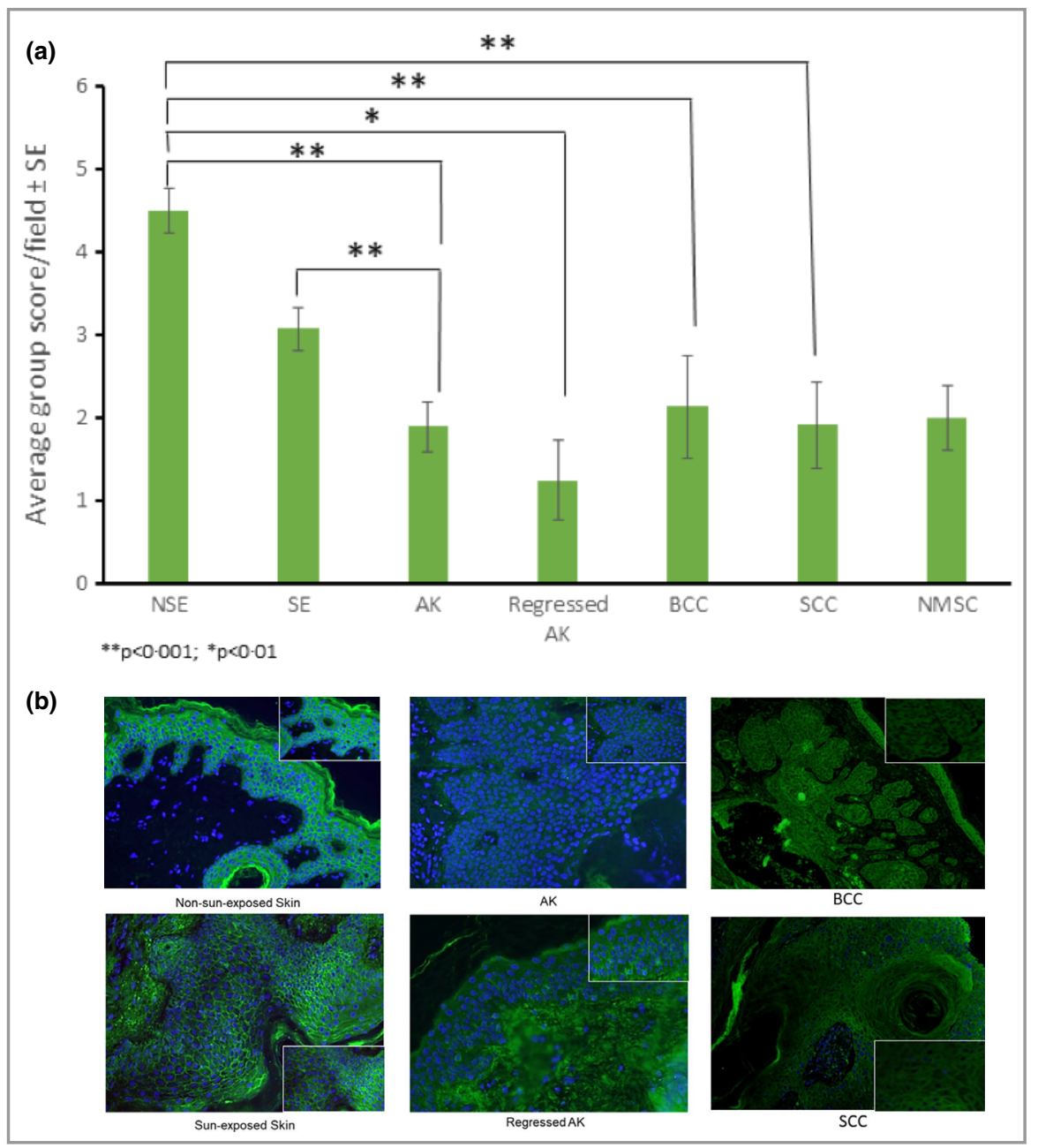

Fig 3. (a) Average E-cadherin expression in skin biopsy samples. Non-sun-exposed skin (NSE), sun-exposed skin (SE), clinically present actinic keratosis (AK), regressed AK, basal cell carcinoma (BCC), squamous cell carcinoma (SCC) and combined BCC and SCC (i.e. nonmelanoma skin cancer, NMSC). (b) Immunofluorescence of E-cadherin with 4',6-diamidino-2-phenylindole. Note that the cell membrane staining that outlines the cells is greater in the NSE and SE than in the AK, regressed AK, BCC and SCC. 


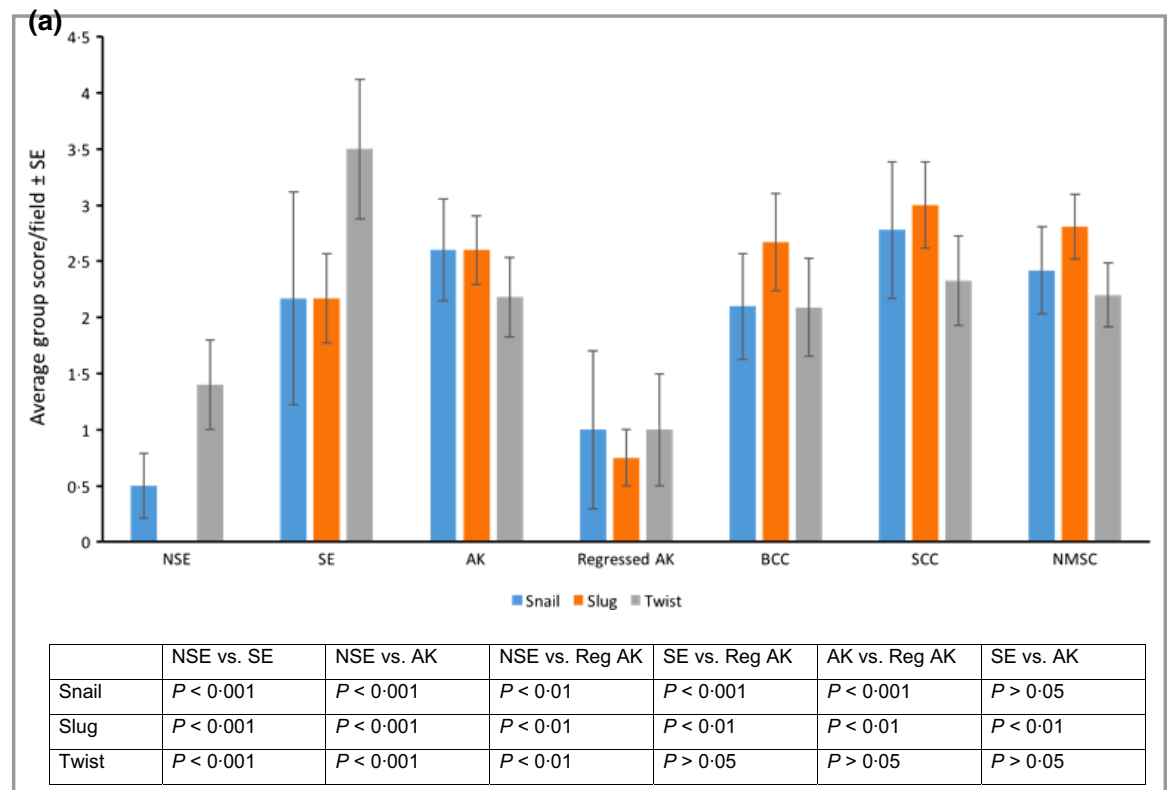

(b)
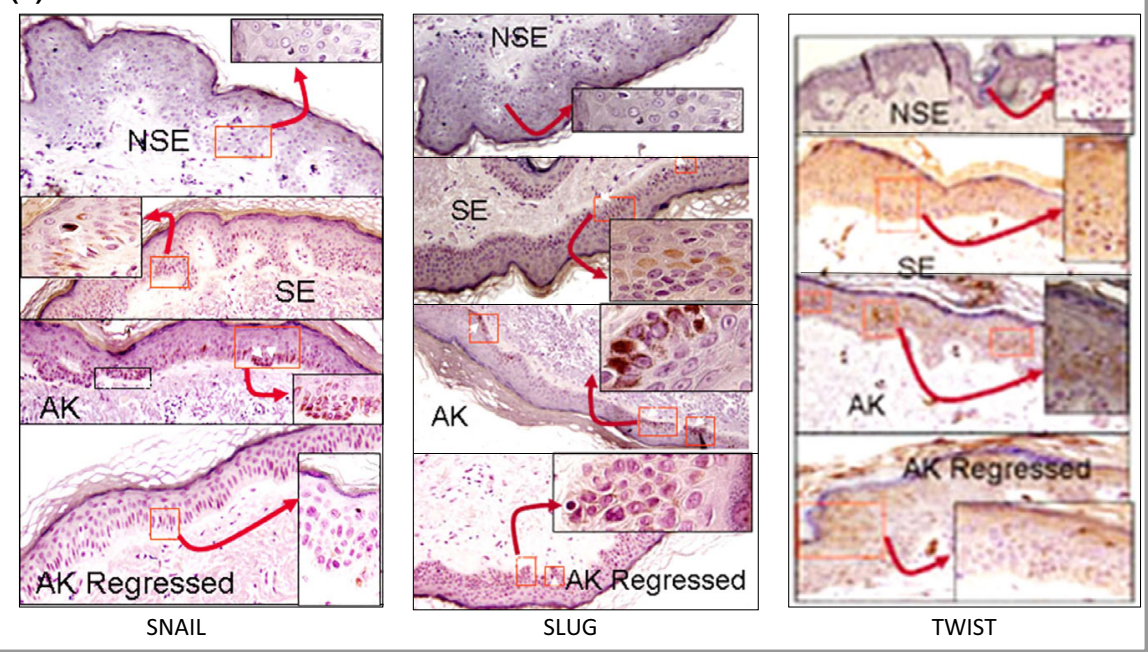

Fig 4. (a) Average group score of Snail, Slug and Twist expression in non-sun-exposed skin (NSE), sun-exposed skin (SE), clinically present actinic keratosis (AK), regressed AK, basal cell carcinoma (BCC), squamous cell carcinoma (SCC) and combined BCC and SCC (i.e. nonmelanoma skin cancer, NMSC). (b) Histochemical staining for Snail, Slug and Twist.

months, suggesting that additional insults are required for them to progress on to become SCCs. In this regard, treatment with the cyclooxygenase-2 inhibitor celecoxib has been shown to diminish the incidence of BCCs and SCCs, but has no effect on AKs. ${ }^{15}$

The percentage of lesions that regressed in our analysis is similar to that reported in some, ${ }^{21}$ but not all studies. ${ }^{22-26}$ This may be due to differences in the extent of actinic damage, the experimental design, regional AK prevalence and/or factors such as patients' age, sex, sunscreen use and/or amount of UV exposure.

There are multiple options for the treatment of AKs, including, but not limited to, local (liquid nitrogen cryotherapy, electrodesiccation and curettage) and field therapy (topical 5fluorouracil, imiquimod, diclofenac, ingenol mebutate and photodynamic therapy). ${ }^{1}$ The concept that AKs can regress and recur has important clinical implications for AK treatment in individuals with extensive actinic damage. As only a minority of AKs are present at any one time, either field treatment or repeated aggressive local treatment is necessary for adequate management of these lesions. Based on the observation that AKs can persist, progress or regress, another implication is that it should be possible to intervene to promote regression and/ or retard progression of AKs.

A main goal of this study was to identify biomarkers that are associated with progression and regression of AKs, in order to develop pharmacological agents that might be employed to inhibit the development of KC. Our analysis found significantly increased p53 expression in AK, BCC, SCC and sun-exposed samples compared with non-sun-exposed 
and regressed AKs. p53 alterations have been associated with the early stages of UV-induced carcinogenesis both in animal models and in human studies. ${ }^{9,27}$ Mutant p53 exhibits a longer half-life than its wild-type counterpart and, as a result, is likely to accumulate in the cell nucleus. ${ }^{28-30} \mathrm{We}$ observed that there was a progressive increase in nuclear p53 staining as the skin progressed from actinically damaged skin to AK to KCs. p53 decreased in regressed AKs. These findings suggest that p53 may be a good biomarker of AK progression. While it is uncertain whether AKs can progress to BCCs, it is well established that people with large numbers of AKs are at increased risk of developing BCCs. ${ }^{3}$ This association is most likely due to the fact that extensive sun exposure causes both.

Downregulation of E-cadherin expression, which leads to a reduction in intercellular adhesion, has previously been linked to $\mathrm{AK}$ and $\mathrm{KC}$ progression. ${ }^{31,32}$ In contrast to the increased expression of p53 with more advanced premalignancies and malignancies, there was a progressive decrease in E-cadherin from non-sun-exposed skin to sun-exposed skin to AKs. Although it was significantly less than in non-sun-exposed skin, it is important to note that there was no further decline in E-cadherin from AKs to NMSCs, implying that loss of Ecadherin may be required for clinical progression of normal skin to sun-damaged skin to AKs, but not for further progression to NMSCs.

Snail, Slug and Twist contribute to the downregulation of E-cadherin and consequently have been connected to invasion and metastasis of cancer cells. ${ }^{11,33}$ Various studies have suggested that such transcriptional repressors are involved in KCs as well, ${ }^{34-36}$ but none has examined the implications of Snail, Slug and Twist in UV-exposed, AK and regressed AK samples. There were higher levels of these proteins in UV-exposed skin and in AKs than in control non-UV-exposed skin. The corresponding reduction of E-cadherin in these samples suggests that increased activity of these transcriptional regulators in AKs and KCs contributes to the loss of E-cadherin and ultimately to the progression of sun-exposed skin to UV-induced cutaneous tumours.

One limitation of our study was that biomarkers were not a primary end point of the study. In addition, for ethical reasons, all patients were allowed to use sunscreen, which may itself alter the natural history of AKs.

An estimated 5 million new cases of NMSC will be treated in the U.S.A. this year. ${ }^{37}$ While the mortality rate for most NMSCs is not high, they can be locally destructive and their treatment represents a tremendous economic impact on the U.S. healthcare system and healthcare systems around the world. ${ }^{5}$ Current methods for their prevention (AK treatment, limiting sun and tanning bed exposure, sunscreens and other photoprotective measures) have not been successful in stemming the increasing incidence of these types of malignancy. Thus, there has been great interest in identifying novel agents for chemoprevention. By examining changes in the expression of p53 and E-cadherin, the findings from this study can be employed to determine the potential efficacy of existing and novel measures for the prevention of NMSCs. Moreover, p53, E-cadherin or one of the E-cadherin repressors could be used as a biomarker to identify subsets of AKs that are at high risk of progressing to SCCs. Our results also confirm that p53 and E-cadherin are relevant targets for the prevention of skin cancer. In this regard, preclinical studies from our laboratory have shown that CP-31398, a pharmacological agent that produces conformational changes in mutant p53, enhancing its biological activity, confers resistance to UV-induced cancer development when applied topically. ${ }^{38}$ Additional therapeutic strategies that enhance E-cadherin expression or lower mutant p53 levels may be effective in reversing the rising incidence of $\mathrm{KC}$.

\section{References}

1 Duncan KO, Geisse JK, Leffell DJ. Epithelial precancerous lesions. In: Fitzpatrick's Dermatology in General Medicine (Goldsmith LA, Katz SI, Gilchrest B, Paller AS, Leffell DJ, Wolff K, eds), 8th edn, Vol. 1. New York: McGraw-Hill, 2012; 1261-83.

2 Rossi R, Mori M, Lotti T. Actinic keratosis. Int J Dermatol 2007; 46:895-904.

3 Foote JA, Harris RB, Giuliano AR et al. Predictors for cutaneous basal- and squamous-cell carcinoma among actinically damaged adults. Int J Cancer 2001; 95:7-11.

4 Neidecker MV, Davis-Ajami ML, Balkrishnan R, Feldman SR. Pharmacoeconomic considerations in treating actinic keratosis. Pharmacoeconomics 2009; 27:451-64.

5 Lim HW, Collins SAB, Resneck JS Jr et al. The burden of skin disease in the United States. J Am Acad Dermatol 2017; 76:958-72.

6 Gallagher RP, Hill GB, Bajdik CD et al. Sunlight exposure, pigmentation factors, and risk of nonmelanocytic skin cancer. II. Squamous cell carcinoma. Arch Dermatol 1995; 131:164-9.

7 Gallagher RP, Hill GB, Bajdik CD et al. Sunlight exposure, pigmentary factors, and risk of nonmelanocytic skin cancer. I. Basal cell carcinoma. Arch Dermatol 1995; 131:157-63.

8 Siegel JA, Korgavkar K, Weinstock MA. Current perspective on actinic keratosis: a review. Br J Dermatol 2017; 177:350-8.

9 Ziegler A, Jonason A, Leffell D et al. Sunburn and p53 in the onset of skin cancer. Nature 1994; 372:773-6.

10 Queille S, Luron L, Spatz A et al. Analysis of skin cancer risk factors in immunosuppressed renal transplant patients shows high levels of UV-specific tandem CC to TT mutations of the p53 gene. Carcinogenesis 2007; 28:724-31.

11 Thiery JP. Epithelial-mesenchymal transitions in tumour progression. Nat Rev Cancer 2002; 2:442-54.

12 Kalluri R, Weinberg RA. The basics of epithelial-mesenchymal transition. J Clin Invest 2009; 119:1420-8.

13 Barrette K, Van Kelst S, Wouters J et al. Epithelial-mesenchymal transition during invasion of cutaneous squamous cell carcinoma is paralleled by AKT activation. Br J Dermatol 2014; 171:1014-21.

14 Moreno-Bueno G, Cubillo E, Sarrio D et al. Genetic profiling of epithelial cells expressing E-cadherin repressors reveals a distinct role for Snail, Slug, and E47 factors in epithelial-mesenchymal transition. Cancer Res 2006; 66:9543-56.

15 Elmets CA, Viner JL, Pentland AP et al. Chemoprevention of nonmelanoma skin cancer with celecoxib: a randomized, doubleblind, placebo-controlled trial. J Natl Cancer Inst 2010; 102:183544.

16 van Roy F, Berx G. The cell-cell adhesion molecule E-cadherin. Cell Mol Life Sci 2008; 65:3756-88. 
17 Green A, Battistutta D. Incidence and determinants of skin cancer in a high-risk Australian population. Int J Cancer 1990; 46:356-61.

18 Karagas MR, Stukel TA, Greenberg ER et al. Risk of subsequent basal cell carcinoma and squamous cell carcinoma of the skin among patients with prior skin cancer. Skin Cancer Prevention Study Group. JAMA 1992; 267:3305-10.

19 English DR, Armstrong BK, Kricker A et al. Demographic characteristics, pigmentary and cutaneous risk factors for squamous cell carcinoma of the skin: a case-control study. Int J Cancer 1998; 76:628-34.

20 Weinstock MA, Thwin SS, Siegel JA et al. Chemoprevention of basal and squamous cell carcinoma with a single course of fluorouracil, 5\%, cream: a randomized clinical trial. JAMA Dermatol 2018; 154: 167-74.

21 Thompson SC, Jolley D, Marks R. Reduction of solar keratoses by regular sunscreen use. N Engl J Med 1993; 329:1147-51.

22 Werner RN, Sammain A, Erdmann R et al. The natural history of actinic keratosis: a systematic review. Br J Dermatol 2013; 169:502-18.

23 Frost C, Williams G, Green A. High incidence and regression rates of solar keratoses in a Queensland community. J Invest Dermatol 2000; 115:273-7.

24 Marks R, Foley P, Goodman G et al. Spontaneous remission of solar keratoses: the case for conservative management. Br J Dermatol 1986; 115:649-55.

25 Harvey I, Frankel S, Marks R et al. Non-melanoma skin cancer and solar keratoses II analytical results of the South Wales Skin Cancer Study. Br J Cancer 1996; 74:1308-12.

26 Criscione VD, Weinstock MA, Naylor MF et al. Actinic keratoses: natural history and risk of malignant transformation in the Veterans Affairs Topical Tretinoin Chemoprevention Trial. Cancer 2009; 115:2523-30.

27 Ziegler A, Leffell DJ, Kunala S et al. Mutation hotspots due to sunlight in the p53 gene of nonmelanoma skin cancers. Proc Natl Acad Sci U S A 1993; 90:4216-20.
28 Giglia-Mari G, Sarasin A. TP53 mutations in human skin cancers. Hum Mutat 2003; 21:217-28.

29 Barzilai A, Lyakhovitsky A, Trau H et al. Expression of p53 in the evolution of squamous cell carcinoma: correlation with the histology of the lesion. J Am Acad Dermatol 2007; 57:669-76.

30 Hall PA, McKee PH, Menage HD et al. High levels of p53 protein in UV-irradiated normal human skin. Oncogene 1993; 8:203-7.

31 Lyakhovitsky A, Barzilai A, Fogel M et al. Expression of E-cadherin and beta-catenin in cutaneous squamous cell carcinoma and its precursors. Am J Dermatopathol 2004; 26:372-8.

32 Papadavid E, Pignatelli $M$, Zakynthinos $S$ et al. Abnormal immunoreactivity of the E-cadherin/catenin $(\alpha-, \beta$-, and $\gamma-)$ complex in premalignant and malignant non-melanocytic skin tumours. J Pathol 2002; 196:154-62.

33 Wang SP, Wang WL, Chang YL et al. p53 controls cancer cell invasion by inducing the MDM2-mediated degradation of Slug. Nat Cell Biol 2009; 11:694-704.

34 Chen H, Takahara M, Xie L et al. Levels of the EMT-related protein Snail/Slug are not correlated with p53/p63 in cutaneous squamous cell carcinoma. J Cutan Pathol 2013; 40:651-6.

35 Toll A, Masferrer E, Hernandez-Ruiz ME et al. Epithelial to mesenchymal transition markers are associated with an increased metastatic risk in primary cutaneous squamous cell carcinomas but are attenuated in lymph node metastases. J Dermatol Sci 2013; 72:93102.

36 Herfs M, Hubert P, Suarez-Carmona M et al. Regulation of p63 isoforms by snail and slug transcription factors in human squamous cell carcinoma. Am J Pathol 2010; 176:1941-9.

37 Rogers HW, Weinstock MA, Feldman SR et al. Incidence estimate of nonmelanoma skin cancer (keratinocyte carcinomas) in the U.S. population, 2012. JAMA Dermatol 2015; 151:1081-6.

38 Tang X, Zhu Y, Han L et al. CP-31398 restores mutant p53 tumor suppressor function and inhibits UVB-induced skin carcinogenesis in mice. J Clin Invest 2007; 117:3753-64. 